\title{
STATEMENT OF PRIORITY
}

The cubically convergent iteration method for the simultaneous determination of all zeros of a polynomial, proposed by 0 . Aberth in [1], is identical to an iterative scheme described earlier by L. W. Ehrlich [2]. The editors, and the author, are indebted to Abdel-Wahab M. Nourein for bringing the earlier reference to their attention.

1. O. ABERTH, "Iteration methods for finding all zeros of a polynomial simultaneously," Math. Comp., v. 27, 1973, pp. 339-344.

2. L. W. EHRLICH, "A modified Newton method for polynomials," Comm. ACM, v. 10, 1967, pp. 107-108.

W. G. 